\title{
Continuous vancomycin in a pediatric cystic fibrosis patient
}

\author{
Cameron J. McKinzie PharmD, BCPPS, BCPS CPP ${ }^{1}$ \\ Charles R. Esther MD, $\mathrm{PhD}^{2}$ (iD) | Timothy J. Vece $\mathrm{MD}^{2}$
}

\author{
${ }^{1}$ Department of Pharmacy, University of \\ North Carolina Medical Center, Chapel Hill, \\ North Carolina \\ 2 Division of Pediatric Pulmonology, \\ Department of Pediatrics, University of North \\ Carolina School of Medicine, Chapel Hill, \\ North Carolina \\ Correspondence \\ Cameron J. McKinzie, PharmD, BCPPS, BCPS, \\ CPP, Department of Pharmacy, University of \\ North Carolina Medical Center, 101 Manning \\ Drive, CB 7600, Chapel Hill 27516, NC. \\ Email: cameron.mckinzie@unchealth.unc.edu
}

\begin{abstract}
Continuous vancomycin has been previously reported to maximize antimicrobial activity while avoiding toxicities associated with dose escalation, but the efficacy of this dosing strategy has not been reported. This case report describes the successful use of continuous vancomycin, including improvement in lung function and avoidance of nephrotoxicity, demonstrated in a pediatric cystic fibrosis (CF) patient with MRSA.

KEYWORDS

cystic fibrosis, MRSA, vancomycin
\end{abstract}

\section{1 | BACKGROUND}

Vancomycin is a glycopeptide antibiotic that is effective against grampositive organisms, including methicillin-resistant Staphylococcus aureus (MRSA). Based on its time-dependent antimicrobial activity, maximal bactericidal effects occur when the concentration of vancomycin remains significantly above the bacterial minimum inhibitory concentration (MIC). However, targeting higher peak concentrations to maximize time above MIC is associated with more adverse effects, such as nephrotoxicity. Continuous vancomycin therapy has been previously reported to maximize the antimicrobial effects of vancomycin while avoiding the toxicities associated with dose escalation and elevated peak concentrations, although the efficacy of this approach was not reported. ${ }^{1}$ Here we describe the successful use of continuous vancomycin in a pediatric cystic fibrosis (CF) patient with MRSA who had a significant drug allergy profile.

\section{2 | CASE}

A 12-year-old girl with CF was admitted to the inpatient pediatric pulmonary service for treatment of a CF pulmonary exacerbation. Her pulmonary function tests (PFTs) on admission were percent predicted (pp) FVC 81, ppFEV 1 79\%, ppFEF $_{25-75} 77 \%$, as compared to her best PFTs in the past year of ppFVC 110, ppFEV 1 105, and $\mathrm{ppFEF}_{25-75}$ 100. Since presenting to our CF center one and a half years prior, the patient has had three bronchoscopy cultures and 12 sputum cultures obtained. Of these, two bronchoscopies were positive for MRSA only, and the third grew MRSA and mold. Of the sputum cultures, eight grew only MRSA, and another (the first obtained at our center) grew Pseudomonas in addition to MRSA.

Given this history of infection primarily with MRSA, prior to admission she was treated with a 3 week course of tedizolid directed against MRSA as her typical respiratory pathogen. On week prior to admission oral minocycline was added to her antibiotic regimen. She underwent bronchoscopy upon admission to determine if there were any other pathogens present that could account for her failure to respond to tedizolid, but cultures only grew $800000 \mathrm{CFU} / \mathrm{mL}$ of MRSA. The patient has multiple allergies and reactions to medications that complicated antibiotic management, including significant hives with both minocycline and ceftaroline, independent of one another; facial swelling with aztreonam; paresthesias with linezolid during two treatment courses; and significant red man syndrome with intermittent dosing of vancomycin. Her most recent culture susceptibilities indicated sensitivity to ceftaroline, doxycycline, linezolid, and vancomycin (with a MIC of $2 \mathrm{mcg} / \mathrm{mL}$ ) and resistance to clindamycin and sulfamethoxazole/trimethoprim.

Upon admission she was started on IV minocycline, however, she developed hives. The pediatric allergy service was consulted and they determined that she was having a delayed hypersensitivity reaction to minocycline, therefore it was discontinued. Given this patient's numerous drug allergies and reactions, the decision was made to treat her pulmonary exacerbation with vancomycin, utilizing a longer infusion time of $2 \mathrm{~h}$ and premedication with diphenhydramine $25 \mathrm{mg}$ to minimize reaction. Despite these interventions, the patient continued to experience intolerable red man symptoms, which did not resolve by increasing the infusion time to $3 \mathrm{~h}$ and diphenhydramine dose to $50 \mathrm{mg}$. After 3 days, she was started on continuous vancomycin, with a goal concentration of $20-25 \mathrm{mcg} / \mathrm{mL}$. The 
TABLE 1 Laboratory values and clinical decision making

\begin{tabular}{lllll} 
Day & BUN $(\mathrm{mg} / \mathrm{dL})$ & $\mathrm{SCr}(\mathrm{mg} / \mathrm{dL})$ & $\begin{array}{l}\text { Vancomycin } \\
\text { concentration }(\mu \mathrm{g} / \mathrm{mL})\end{array}$ & Action taken \\
\hline 1 & 10 & 0.42 & - & - \\
\hline 8 & 11 & 0.45 & 25.4 & Continued same dose \\
\hline 9 & - & - & 27.7 & $\begin{array}{l}\text { Decreased dose to } 2500 \mathrm{mg} / \mathrm{day} \\
(53 \mathrm{mg} / \mathrm{kg})\end{array}$ \\
\hline 10 & 10 & 0.46 & 21.8 & Continued same dose \\
\hline 11 & 8 & 0.46 & 23.2 & Continued same dose \\
\hline $14^{\mathrm{a}}$ & 10 & 0.45 & - & - \\
\hline
\end{tabular}

${ }^{a}$ These labs were obtained while the patient was outpatient.

patient was initiated on $3000 \mathrm{mg}$ over $24 \mathrm{~h}(64 \mathrm{mg} / \mathrm{kg})$, and a level was obtained the following morning, about $22.5 \mathrm{~h}$ after the start of the infusion (Table 1). The patient remained in the hospital for five additional days after starting the continuous vancomycin, during which time one dose adjustment was made (Table 1). Prior to discharge, her repeat PFTs demonstrated significant improvement in her lung function to $\mathrm{ppFEV}_{1} 103$. She was discharged home, where she completed an additional 5 days of continuous vancomycin before discontinuing vancomycin due to development of hives on her lower extremities. No changes in renal function were observed, and the patient felt that the red man symptoms she experienced were tolerable with diphenhydramine $25 \mathrm{mg}$ as needed.

\section{3 | DISCUSSION}

The evidence supporting the use of continuous vancomycin is limited. The majority of studies evaluating continuous vancomycin are in adult patients, and most evaluate nephrotoxicity and adverse effects, as opposed to clinical efficacy, as the primary endpoint. ${ }^{2-3}$ These previous studies have demonstrated that continuous vancomycin does not result in an increased incidence of nephrotoxicity, and in some studies, the incidence was significantly less when compared to intermittent vancomycin.

In 2012, McKamy et al, ${ }^{1}$ described a dosing guideline that converted pediatric patients at a single institution from intermittent vancomycin dosing to continuous vancomycin for patients who had two consecutive subtherapeutic trough concentrations. Patients had to have either pneumonia or osteomyelitis due to gram-positive organisms, including MRSA. The authors found few adverse effects associated when patients were transitioned to continuous vancomycin, including nephrotoxicity. This study did not report patient outcomes.

In our patient, it is interesting to note that she tolerated continuous vancomycin despite her significant red man syndrome with intermittent dosing. Given that red man syndrome is typically associated with rapid infusion of vancomycin, it is reasonable to consider that the slower continuous infusion may be more tolerable. ${ }^{4}$

Our patient's case demonstrates successful use of continuous vancomycin in a pediatric CF patient with significant drug allergies related to antimicrobials targeting her MRSA, as well as resistance limiting therapeutic options. In addition to achieving therapeutic vancomycin concentrations from the beginning of therapy, our patient also had no nephrotoxicity, as assessed by serum creatinine, associated with the continuous vancomycin and had a significant improvement in PFTs, demonstrating efficacy of this dosing regimen.

\section{CONFLICTS OF INTEREST}

The views expressed in this article do not communicate an official position of the University of North Carolina Medical Center or University of North Carolina School of Medicine.

\section{ORCID}

Cameron J. McKinzie (iD http://orcid.org/0000-0003-1401-5628

Charles R. Esther (iD) http://orcid.org/0000-0002-8081-2986

\section{REFERENCES}

1. McKamy S, Chen T, Lee M, Ambrose PJ. Evaluation of a pediatric continuous-infusion vancomycin guideline. Am J Health-Syst Pharm. 2012;69:2066-2071.

2. Man SSK, Carr RR, Ensom MHH. Comparison of continuous and intermittent IV infusion of vancomycin: systematic review. Can J Hosp Pharm. 2010;63: 373-381.

3. DiMondi VP, Rafferty K. Review of continuous-infusion vancomycin. Ann Pharmacother. 2013;47:219-227.

4. Wallace MR, Mascola JR, Oldfield EC. Red man syndrome: incidence, aetiology and prophylaxis. J Infect Dis. 1991;164:1180-1185.

How to cite this article: McKinzie CJ, Esther CR, Vece TJ. Continuous vancomycin in a pediatric cystic fibrosis patient. Pediatric Pulmonology. 2018;53:E4-E5. https://doi.org/10.1002/ppul.23844 